\title{
¿Utilizarán los futuros docentes las tecnologías móviles? Validación de una propuesta de modelo TAM extendido
}

\section{Will future teachers use mobile technologies? Validation of an extended TAM model proposal}

\author{
José Carlos Sánchez Prieto \\ Universidad de Salamanca, Salamanca. España \\ josecarlos.sp@usal.es \\ Susana Olmos Migueláñez \\ Universidad de Salamanca, Salamanca. España \\ solmos@usal.es \\ Francisco J. García-Peñalvo \\ Universidad de Salamanca, Salamanca. España \\ fgarcia@usal.es
}

\begin{abstract}
Resumen
El desarrollo de modelos de adopción tecnológica para su aplicación dentro del campo educativo constituye una tendencia de creciente interés. Una práctica habitual dentro de estas investigaciones es la aplicación de modelos basados en TAM expandidos con otros constructos. El presente artículo se enmarca dentro de esta línea, presentando una propuesta que analiza los efectos de la resistencia al cambio y la compatibilidad sobre la intención de uso de tecnologías móviles en la futura práctica docente entre los maestros en formación. Con este objetivo se realizó un estudio en el que participaron 678 estudiantes del Grado de Maestro de Primaria de la Universidad de Salamanca y se sometió al modelo a un análisis factorial para confirmar su validez. Los resultados obtenidos muestran una intención de uso favorable hacia el uso de tecnologías móviles por parte de los estudiantes, el contraste de hipótesis reveló algunas diferencias significativas en función del género, curso y centro de pertenencia de los estudiantes y el análisis factorial llevado a cabo reflejó unos adecuados índices de bondad de ajuste, pese a los problemas de validación.
\end{abstract}

Palabras clave: TAM, SEM, aprendizaje móvil, maestros en formación, aceptación tecnológica.

\begin{abstract}
The development of technology acceptance models for their implementation in the field of education constitutes an increasingly interesting trend. A common practice in this kind of research is to develop TAM-based models expanded with other constructs. This paper belongs to this line, presenting a proposal analyzing the effects of resistance to change and compatibility on the intention of using mobile technologies in pre-service teachers' future practice. To this end, we conducted a study with 678 students from the Primary Education Teacher Bachelor's Degree from the University of Salamanca, and we subjected the model to a factorial analysis to confirm its validity. The results show the students' favorable intention of using mobile technologies. The hypothesis test revealed some significant differences according to student gender, year and school, and the factor analysis we carried out reflected adequate goodness of fit indices despise some validity issues.
\end{abstract}

Keywords: TAM, SEM, mobile learning, pre-service teachers, technological acceptance. 


\section{Introducción}

La inclusión de las TIC en los centros escolares constituye un problema cada vez más acuciante, empujado tanto por la necesidad de que las aulas se adapten y reflejen los cambios sociales que el rápido desarrollo tecnológico está trayendo consigo, como por los beneficios y posibilidades didácticas, inherentes a los nuevos sistemas de información, que pueden contribuir a la mejora del proceso de enseñanza aprendizaje.

Entre las nuevas metodologías surgidas recientemente destaca el aprendizaje móvil, o mobile learning, que busca explotar las posibilidades didácticas de los dispositivos móviles, contribuyendo a la flexibilización y personalización del proceso educativo (Sánchez Prieto, Olmos Migueláñez y García-Peñalvo, 2014).

Dentro del proceso de integración tecnológico podemos encontrar tres agentes cuya colaboración resulta fundamental para que este se consolide con éxito: los alumnos, las administraciones públicas y los profesores (Chen, Looi y Chen, 2009).

Conocer la actitud de los docentes hacia una tecnología determinada puede resultar una herramienta muy útil a la hora de predecir el éxito o fracaso de la incorporación de un nuevo sistema de información (SI). Así mismo, determinar los factores clave que afectan al uso real de un SI por parte de este colectivo puede servir de guía para orientar el proceso de integración evitando posibles errores (Fong et al., 2014).

Los modelos de adopción tecnológica suponen una alternativa eficaz para el estudio del proceso que lleva al individuo a utilizar las nuevas tecnologías.

La más popular de estas teorías es el TAM (Modelo de Aceptación Tecnológica). Enunciado por Davis (1989), este modelo se elabora partiendo de los supuestos de la TRA (Teoría de la Acción Razonada) (Fishbein y Ajzen, 1975) y la TPB (Teoría del Comportamiento Planeado) (Ajzen, 1985), dos teorías procedentes del ámbito de la psicología cognitiva, que analizan el proceso que lleva a un sujeto a adoptar un comportamiento determinado.

El TAM parte de dos conceptos básicos:

- Utilidad Percibida (PU): El grado en el que un individuo percibe que el uso de la herramienta puede aumentar su eficacia en el desempeño de una tarea

- Facilidad de uso percibida (PEU): La percepción del sujeto de la cantidad de esfuerzo necesario para el uso de la tecnología.

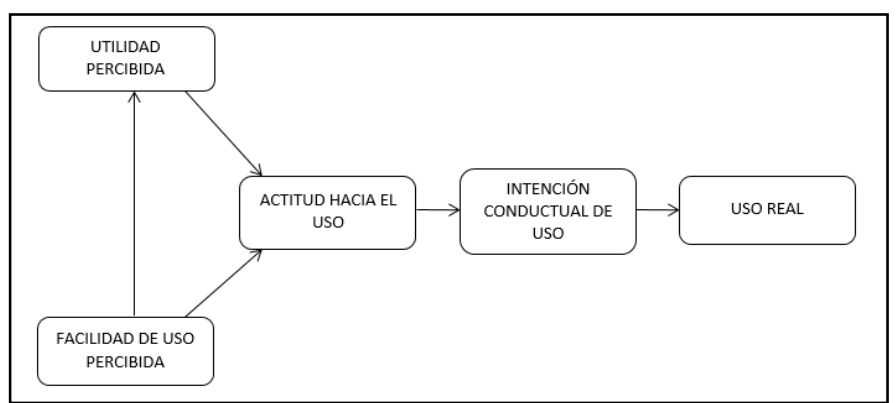

Figura. 1. Esquema del modelo TAM (Davis, Bagozzi y Warshaw, 1989).

¿Utilizarán los futuros docentes las tecnologías móviles? Validación de una propuesta de modelo TAM extendido. José Carlos Sánchez Prieto, Susana Olmos Migueláñez y Francisco José García-Peñalvo.

Página 2 de 
Estos dos factores influyen en tres constructos ya presentes en la teoría de Fishbein y Ajzen: la actitud hacia el uso de la tecnología (A), compuesta por los valores y creencias del individuo sobre el uso; la intención conductual de uso (BI), condicionada por la actitud y definida como la probabilidad de que el sujeto utilice el sistema y, finalmente, el uso real (AU) (Figura 1).

Las principales ventajas de esta teoría son su sencillez, adaptabilidad y solidez teórica, tratándose del modelo de aceptación más empleado en la actualidad (King y He, 2006). Aplicado en numerosos ámbitos (Ariff, Yeow y Zakuan, 2014; Briz-Ponce y GarcíaPeñalvo, 2015), se modifica frecuentemente añadiendo constructos de otras teorías como la TTF (Task Technology Fit) (Ma, Chao y Cheng, 2013) o la IDT (Innovation Diffusion Theory) (Lee, Hsieh y Hsu, 2011). También, con el tiempo, el modelo TAM ha ido incorporando algunos de los hallazgos de estas investigaciones generándose dos versiones del modelo el TAM2 (Venkatesh y Davis, 2000) y el TAM3 (Venkatesh y Bala, 2008), que plantean nuevos constructos y relaciones, aunque también incrementan la complejidad del modelo.

En el contexto educativo, se pueden encontrar ejemplos de su uso a nivel nacional (Parra-Meroño y Carmona-Martínez, 2011; Sánchez-Franco, 2010; Sánchez-Franco, Martínez-López y Martín-Velicia, 2009) e internacional, para estudiar tanto las actitudes de los estudiantes (Abbad, 2011; Bachtiar, Rachmadi y Pradana, 2014), como las de los profesores (Rienties, Giesbers, Lygo-Baker, Ma y Rees, 2016; Wang y Wang, 2009).

Nuestra investigación se enmarca dentro de los estudios que aplican el modelo con maestros en su etapa de formación universitaria, realizados con la finalidad de analizar la intención de uso de nuevas tecnologías en su futuro ejercicio docente.

Este artículo tiene como objetivo presentar los resultados de un estudio sobre la aceptación de las tecnologías móviles entre los estudiantes del Grado de Maestro de Primaria centrado en la influencia de la compatibilidad y la resistencia al cambio. Para ello proponemos una estructura dividida en tres secciones. La primera de ellas está destinada a describir la metodología. En ella se detalla la composición del modelo y sus variables, así como la muestra y el instrumento elaborado para la recogida de datos. En la segunda sección se exponen los resultados obtenidos, incluyendo el análisis factorial realizado para validar el instrumento y el contraste de hipótesis para determinar la influencia del género, curso y centro educativo. Por último, finalizaremos el artículo con unas breves conclusiones.

\section{Metodología}

Nuestra propuesta plantea un problema de investigación relacionado con los factores que conducen al uso de tecnologías móviles por parte de los docentes.

El objetivo de la investigación es conocer el nivel de aceptación de las tecnologías móviles entre los maestros en formación, entendiendo como tal la intención de uso de dichos dispositivos en su futura práctica docente.

Esta sección expone la metodología de investigación empleada. Con este fin, se comienza describiendo el modelo teórico, definiendo los constructos añadidos al modelo y las hipótesis relacionales planteadas. Tras esto, se presentan las variables y la

¿Utilizarán los futuros docentes las tecnologías móviles? Validación de una propuesta de modelo TAM extendido. José Carlos Sánchez Prieto, Susana Olmos Migueláñez y Francisco José García-Peñalvo.

Página 3 de 
población y muestra del estudio. Por último, se detalla el instrumento empleado para la recogida de datos.

\section{Modelo de Investigación}

El presente modelo de investigación está compuesto por tres constructos provenientes del modelo TAM, a los que decidimos añadir un factor habilitador, la compatibilidad percibida, y otro inhabilitante, la resistencia al cambio, para estudiar su papel en el proceso de aceptación tecnológica.

\section{Constructos del modelo TAM}

El punto de partida para el desarrollo de nuestro esquema teórico es el modelo TAM de Davis, del que hemos incluido los constructos: facilidad de uso percibida, utilidad percibida e intención conductual. Para este estudio hemos eliminado el constructo actitud hacia el uso para centrarnos en el estudio de la relación entre la utilidad y la facilidad de uso percibida y la intención conductual, simplificando de esta manera tanto el modelo como el instrumento (Davis y Venkatesh, 1996).

El constructo uso real ha sido también eliminado, dado que se trata de una investigación sobre la intención de uso futuro de los dispositivos. Esto también tiene su precedente en otros estudios con esta población (Acarli y Sağlam, 2015; Teo y Noyes, 2011; Valtonen et al., 2015).

Las relaciones entre estos tres constructos han sido analizadas en un amplio número de investigaciones dentro del ámbito educativo (Tabla 1), estudiándose tanto las relaciones entre ellos, como con otras dimensiones procedentes de otros modelos y teorías.

Tabla. 1. Relaciones entre los constructos procedentes del TAM analizadas en investigaciones relevantes.

\begin{tabular}{|c|c|}
\hline \multicolumn{2}{|c|}{ Park (2009) } \\
\hline $\mathrm{PU} \rightarrow \mathrm{BI}$ & No soportada \\
\hline $\mathrm{PEU} \rightarrow \mathrm{BI}$ & No soportada \\
\hline $\mathrm{PEU} \rightarrow \mathrm{PU}$ & Soportada \\
\hline \multicolumn{2}{|c|}{ Abbad, Morris y Nahlik (2009) } \\
\hline $\mathrm{PU} \rightarrow \mathrm{BI}$ & Soportada \\
\hline $\mathrm{PEU} \rightarrow \mathrm{BI}$ & Soportada \\
\hline $\mathrm{PEU} \rightarrow \mathrm{PU}$ & Soportada \\
\hline \multicolumn{2}{|c|}{ Chen, Lin, Yen y Lou (2013) } \\
\hline $\mathrm{PU} \rightarrow \mathrm{BI}$ & Soportada \\
\hline $\mathrm{PEU} \rightarrow \mathrm{BI}$ & Soportada \\
\hline $\mathrm{PEU} \rightarrow \mathrm{PU}$ & Soportada \\
\hline \multicolumn{2}{|c|}{ Venter, van Rensburg y Davis (2012 } \\
\hline $\mathrm{PU} \rightarrow \mathrm{BI}$ & Soportada \\
\hline $\mathrm{PEU} \rightarrow \mathrm{BI}$ & Soportada \\
\hline $\mathrm{PEU} \rightarrow \mathrm{PU}$ & Soportada \\
\hline \multicolumn{2}{|c|}{ Huang, Liaw y Lai (2013) } \\
\hline $\mathrm{PU} \rightarrow \mathrm{BI}$ & Soportada \\
\hline $\mathrm{PEU} \rightarrow \mathrm{BI}$ & Soportada \\
\hline \multicolumn{2}{|c|}{ Mejia y Phelan (2014) } \\
\hline
\end{tabular}

¿Utilizarán los futuros docentes las tecnologías móviles? Validación de una propuesta de modelo TAM extendido. José Carlos Sánchez Prieto, Susana Olmos Migueláñez y Francisco José García-Peñalvo.

Página 4 de 


\begin{tabular}{|l|l|}
\hline PUBI & No soportada \\
PEUBI & Soportada \\
PEUPU & Soportada \\
\hline
\end{tabular}

Para los constructos procedentes del modelo TAM se plantean las siguientes hipótesis, partiendo de las planteadas por Davis en su propuesta original:

- H1: La utilidad percibida está positivamente relacionada con la intención de uso de tecnologías móviles de los estudiantes del grado de maestro de educación primaria en su futura práctica docente.

- H2: La facilidad de uso percibida está positivamente relacionada con la intención de uso de tecnologías móviles de los estudiantes del grado de maestro de educación primaria en su futura práctica docente.

- H3: La facilidad de uso percibida está positivamente relacionada con la utilidad percibida por los estudiantes en el uso de tecnologías móviles en su futura práctica docente.

\section{Compatibilidad percibida}

La compatibilidad percibida es un constructo procedente de la teoría de la difusión de innovaciones (IDT) (Rogers, 1962) y se encuentra dentro de los denominados factores motivacionales extrínsecos, que engloban a aquellos que son causados por presiones externas a la persona (Hernández García, 2008). Dentro de este grupo se encuentran también otros factores, como la utilidad o la facilidad de uso percibida, bases del TAM, por lo que consideramos interesante añadir otros constructos pertenecientes al mismo grupo para analizar sus posibles efectos explicativos y la relación entre ellos.

La IDT propone cinco características tecnológicas clave que ayudan a explicar la tasa de adopción por parte de los usuarios: la ventaja relativa sobre las tecnologías precedentes, la complejidad de uso, la posibilidad de prueba, la posibilidad de observación de resultados y la compatibilidad con las características de los usuarios.

Rogers define la compatibilidad como "el grado en el que una innovación es percibida como consistente con los valores, experiencias pasadas y necesidades de los potenciales adoptantes" (Rogers, 1962 p. 15).

La integración de un nuevo sistema de información trae consigo una carga de valores que, si no coinciden con los valores preexistentes, deben ser adoptados por el cuerpo docente, dificultando por tanto la aceptación.

Aunque la definición de Rogers es la más empleada, existe cierta discrepancia acerca de la parte final de la definición, ya que hay autores que consideran que la compatibilidad con las necesidades del posible usuario tiene más que ver con el concepto de ventaja relativa (Moore y Benbasat, 1991).

Con los años, se ha profundizado en las dimensiones de la compatibilidad con la tecnología. Tornatzky y Klein (1982) proponen dos tipos de compatibilidad:

- Compatibilidad normativa: Relacionada con las preconcepciones y sentimientos de la persona hacia la tecnología.

¿Utilizarán los futuros docentes las tecnologías móviles? Validación de una propuesta de modelo TAM extendido. José Carlos Sánchez Prieto, Susana Olmos Migueláñez y Francisco José García-Peñalvo.

Página 5 de 
- Compatibilidad operacional: Término que hace referencia a la concordancia del sistema de información con los hábitos del sujeto.

En esta misma línea Karahanna, Agarwal y Angst (2006), partiendo de la tipología anterior, dividen la compatibilidad operacional en tres dimensiones aplicadas al entorno laboral. De esta manera proponen la siguiente clasificación:

- Compatibilidad con los valores: Equivalente a la compatibilidad normativa de Tornatzky y Klein.

- Compatibilidad con la experiencia previa: Definida como el ajuste entre la tecnología objeto de estudio y los usos tecnológicos previos del sujeto.

- Compatibilidad con el estilo preferido de trabajo: Hace referencia a la posibilidad de que la tecnología sea consistente con la forma de trabajar deseada por la persona.

- Compatibilidad con las prácticas existentes: Recoge la manera en que el sistema de información encaja en el proceso actual de trabajo del individuo.

Este factor ha sido incorporado con anterioridad en modelos basados en TAM con resultados positivos con estudiantes universitarios, como la conducida por EscobarRodríguez y Monge-Lozano (2012) para analizar la aceptación de Moodle entre los estudiantes del Grado de Administración de Empresas, para ello elaboraron un modelo basado en TAM al que añadieron los constructos de utilidad percibida por los profesores, formación en el manejo y compatibilidad con las tareas de los alumnos. Este último constructo fue desarrollado englobando aspectos de la compatibilidad con el estilo preferido de trabajo y compatibilidad con las prácticas existentes, los resultados del estudio soportaron la relación entre la compatibilidad y la facilidad de uso.

También sobre esta temática encontramos investigaciones como la de Najmul Islam (2013), en la que se plantea el efecto de la compatibilidad no como antecedente del uso de sistemas eLearning (García-Peñalvo y Seoane-Pardo, 2015), sino como variable moderadora entre el uso y los beneficios percibidos por los estudiantes, obteniendo buenos resultados.

En cuanto a las relaciones entre la compatibilidad y otros constructos, lo más frecuente es situarla como antecedente de la intención conductual de uso y la utilidad percibida (Sun, Bhattacherjee y Ma, 2009). Dentro del campo educativo se sigue esta misma tendencia, aunque con algunas excepciones como las mencionadas anteriormente (Tabla 2).

Tabla. 2. Relaciones entre la compatibilidad percibida y constructos analizadas en investigaciones relevantes.

\begin{tabular}{|c|c|}
\hline \multicolumn{2}{|c|}{ Lee, Hsieh y Hsu (2011) } \\
\hline $\begin{array}{l}\mathrm{PC} \rightarrow \mathrm{PU} \\
\mathrm{PC} \rightarrow \mathrm{PEU} \\
\mathrm{PC} \rightarrow \mathrm{BI}\end{array}$ & $\begin{array}{l}\text { Soportada } \\
\text { No soportada } \\
\text { Soportada }\end{array}$ \\
\hline \multicolumn{2}{|c|}{ Escobar-Rodríguez y Monge-Lozano (2012) } \\
\hline $\begin{array}{l}\mathrm{PC} \rightarrow \mathrm{PU} \\
\mathrm{PC} \rightarrow \mathrm{PEU}\end{array}$ & $\begin{array}{l}\text { No soportada } \\
\text { Soportada }\end{array}$ \\
\hline
\end{tabular}

¿Utilizarán los futuros docentes las tecnologías móviles? Validación de una propuesta de modelo TAM extendido. José Carlos Sánchez Prieto, Susana Olmos Migueláñez y Francisco José García-Peñalvo.

Página 6 de 


\begin{tabular}{|c|c|}
\hline \multicolumn{2}{|c|}{ Stols y Kriek (2011) } \\
\hline $\mathrm{PC} \rightarrow \mathrm{PU}$ & Soportada \\
\hline \multicolumn{2}{|c|}{ Zhang y Xu (2011) } \\
\hline $\mathrm{PC} \rightarrow$ MMA (mantenimiento de modelos mentales) & Soportada \\
\hline $\mathrm{PC} \rightarrow \mathrm{MMB}$ (construcción de modelos mentales) & Soportada \\
\hline \multicolumn{2}{|c|}{ Chang y Tung (2008) } \\
\hline $\begin{array}{l}\mathrm{PC} \rightarrow \mathrm{PU} \\
\mathrm{PC} \rightarrow \mathrm{PI}\end{array}$ & Soportada \\
\hline \multicolumn{2}{|c|}{ Chen (2011) } \\
\hline $\begin{array}{l}\mathrm{PC} \rightarrow \mathrm{BI} \\
\mathrm{PC} \rightarrow \mathrm{TE} \text { (Expectación tecnológica) }\end{array}$ & $\begin{array}{l}\text { Soportada } \\
\text { Soportada }\end{array}$ \\
\hline \multicolumn{2}{|c|}{ Hung y Cho (2008) } \\
\hline $\mathrm{PC} \rightarrow \mathrm{CU}$ (Continuación de uso) & No soportada \\
\hline \multicolumn{2}{|c|}{ Cao, Ajjan y Hong (2013) } \\
\hline $\mathrm{PC} \rightarrow \mathrm{AU}$ & Soportada \\
\hline \multicolumn{2}{|c|}{ Cheng (2015) } \\
\hline $\begin{array}{l}\mathrm{PC} \rightarrow \mathrm{PE} \text { (Entretenimiento percibido) } \\
\mathrm{PC} \rightarrow \mathrm{PU} \\
\mathrm{PC} \rightarrow \mathrm{PEU} \\
\mathrm{PC} \rightarrow \mathrm{BI}\end{array}$ & $\begin{array}{l}\text { Soportada } \\
\text { Soportada } \\
\text { Soportada } \\
\text { Soportada }\end{array}$ \\
\hline \multicolumn{2}{|c|}{ Šebjan y Tominc (2015) } \\
\hline $\begin{array}{l}\mathrm{PC} \rightarrow \mathrm{BI} \\
\mathrm{PC} \rightarrow \mathrm{PU}\end{array}$ & $\begin{array}{l}\text { Soportada } \\
\text { Soportada }\end{array}$ \\
\hline \multicolumn{2}{|c|}{ John (2015) } \\
\hline $\mathrm{PC} \rightarrow \mathrm{PEU}$ & Soportada \\
\hline \multicolumn{2}{|c|}{ Wu y Liu (2013) } \\
\hline $\mathrm{PC} \rightarrow \mathrm{A}$ & No soportada \\
\hline \multicolumn{2}{|c|}{ Liao y Lu (2008) } \\
\hline $\mathrm{PC} \rightarrow \mathrm{ICU}$ (Intención de continuación de uso) & Soportada \\
\hline
\end{tabular}

Para nuestra propuesta, optamos por plantear las relaciones más habituales formulando, por tanto, las siguientes hipótesis:

- H4: La compatibilidad percibida está positivamente relacionada con la utilidad percibida por los estudiantes en el uso de tecnologías móviles en su futura práctica docente.

- H5: La compatibilidad percibida está positivamente relacionada con la intención de uso de tecnologías móviles de los estudiantes de magisterio en su futura práctica docente.

\section{Resistencia al cambio}

A diferencia de la compatibilidad, la Resistencia al cambio es un constructo relativamente joven cuyo papel en el proceso de aceptación tecnológica está comenzando a ser explorado.

¿Utilizarán los futuros docentes las tecnologías móviles? Validación de una propuesta de modelo TAM extendido. José Carlos Sánchez Prieto, Susana Olmos Migueláñez y Francisco José García-Peñalvo.

Página 7 de 
El origen de este constructo se encuentra ligado al modelo dual de uso de la tecnología (Cenfetelli, 2004). Esta teoría establece dos grupos de factores que recogen las creencias externas del individuo sobre los atributos del sistema que influyen en su aceptación o rechazo. Estos grupos son:

- Factores facilitadores: Término que hace referencia a "aquellas creencias externas relacionadas con el diseño y funcionamiento del sistema que animan o desaniman su uso dependiendo de su valor" (Cenfetelli 2004, p. 475). Dentro de este grupo se situarían constructos como la utilidad, la facilidad de uso o la calidad del sistema.

- Factores inhibidores: A diferencia de los factores facilitadores, los factores inhibidores recogen aquellas creencias externas sobre el sistema que sólo producen un efecto negativo sobre la aceptación. Los factores inhibidores deben cumplir tres condiciones: Los inhibidores no son los opuestos de los facilitadores, los inhibidores y los facilitadores son independientes entre sí y los inhibidores y los facilitadores tienen distintos antecedentes y efectos.

Mientras que el grupo de factores facilitadores está presente en un gran número de investigaciones, los factores inhibidores constituyen un grupo poco analizado (Bhattacherjee y Hikmet, 2007). La resistencia al cambio nace como constructo con la intención de explorar estos factores.

Este término, relacionado con la fuerza que se opone a la discontinuidad del status quo (Lewin, 1947), puede ser definido como la dificultad para romper las rutinas o el estrés emocional producido ante la expectativa de cambios (Guo, Sun, Wang, Peng y Yan, 2013).

Esta definición de resistencia al cambio sitúa al constructo próximo al de compatibilidad percibida, más concretamente a los constructos de compatibilidad con el estilo preferido de trabajo y compatibilidad con las prácticas existentes propuestos por Karahanna, Agarwal y Angst (2006). Sin embargo, conviene precisar que resistencia al cambio no significa lo mismo que incompatibilidad, ya que la resistencia al cambio hace referencia a la oposición a cualquier cambio del status quo sin importar que este sea compatible o no con los valores ideas o experiencias pasadas del usuario potencial.

Aunque no está integrado en ninguna de las grandes teorías, ha sido explorado en estudios de aceptación en campos como la economía (Al-Somali, Gholami y Clegg, 2009) o la medicina (Hsieh, Lai y Ye, 2014), soportándose su relación con constructos como la intención conductual o la utilidad percibida (Tabla 2). Sin embargo, en el campo educativo se trata de una variable muy poco explorada, aunque podemos encontrar algunos ejemplos, como la investigación conducida por Chen, Wang y Lin (2013), que la incluyen en su modelo para explicar la intención de uso de un LMS (Learning Management System) por parte de estudiantes universitarios ofreciendo buenos resultados.

Tabla. 3. Relaciones entre la resistencia al cambio y otros constructos analizadas en investigaciones relevantes.

\begin{tabular}{|l|l|}
\hline \multicolumn{2}{|c|}{ Guo et. al. (2013) } \\
\hline $\begin{array}{l}\mathrm{RC} \rightarrow \mathrm{PU} \\
\mathrm{RC} \rightarrow \mathrm{PEU}\end{array}$ & $\begin{array}{l}\text { Soportada } \\
\text { No soportada }\end{array}$ \\
\hline
\end{tabular}

¿Utilizarán los futuros docentes las tecnologías móviles? Validación de una propuesta de modelo TAM extendido. José Carlos Sánchez Prieto, Susana Olmos Migueláñez y Francisco José García-Peñalvo.

Página 8 de 


\begin{tabular}{|c|c|}
\hline \multicolumn{2}{|c|}{ Al-Somali, Gholami y Clegg (2009) } \\
\hline $\mathrm{RC} \rightarrow \mathrm{A}$ & Soportada \\
\hline \multicolumn{2}{|c|}{ Bhattacherjee y Hikmet (2007) } \\
\hline $\begin{array}{l}\mathrm{RC} \rightarrow \mathrm{BI} \\
\mathrm{RC} \rightarrow \mathrm{AU}\end{array}$ & $\begin{array}{l}\text { Soportada } \\
\text { Soportada }\end{array}$ \\
\hline \multicolumn{2}{|c|}{ Nov y Ye (2009) } \\
\hline $\mathrm{RC} \rightarrow \mathrm{CA}$ (Ansiedad ante los ordenadores) & Soportada \\
\hline $\mathrm{RC} \rightarrow \mathrm{RD}$ (Demostrabilidad de los resultados) & Soportada \\
\hline $\mathrm{RC} \rightarrow \mathrm{EE}$ (Expectativa de esfuerzo) & Soportada \\
\hline \multicolumn{2}{|c|}{ Hsieh (2015) } \\
\hline $\mathrm{RC} \rightarrow \mathrm{BI}$ & Soportada \\
\hline \multicolumn{2}{|c|}{ Hsieh, Lai y Hong (2015) } \\
\hline $\begin{array}{l}\mathrm{RC} \rightarrow \mathrm{BI} \\
\mathrm{BI} \rightarrow \mathrm{RC}\end{array}$ & $\begin{array}{l}\text { Soportada } \\
\text { Soportada }\end{array}$ \\
\hline \multicolumn{2}{|c|}{ Gagnon et. al. (2014) } \\
\hline $\mathrm{RC} \rightarrow \mathrm{BI}$ & Soportada \\
\hline \multicolumn{2}{|c|}{ Nov y Schecter (2012) } \\
\hline $\mathrm{RC} \rightarrow \mathrm{PC}$ & Soportada \\
\hline $\mathrm{RC} \rightarrow \mathrm{PU}$ & Soportada \\
\hline $\mathrm{RC} \rightarrow \mathrm{PEU}$ & Soportada \\
\hline \multicolumn{2}{|c|}{ Chen, Wang y Lin (2013) } \\
\hline $\mathrm{RC} \rightarrow \mathrm{PU}$ & Soportada \\
\hline $\mathrm{RC} \rightarrow \mathrm{BI}$ & Soportada \\
\hline \multicolumn{2}{|c|}{ Biranvand, Hakkak y Nejad (2015) } \\
\hline $\mathrm{PU} \rightarrow \mathrm{RC}$ & Soportada \\
\hline $\mathrm{PEU} \rightarrow \mathrm{RC}$ & Soportada \\
\hline $\mathrm{RC} \rightarrow \mathrm{A}$ & Soportada \\
\hline
\end{tabular}

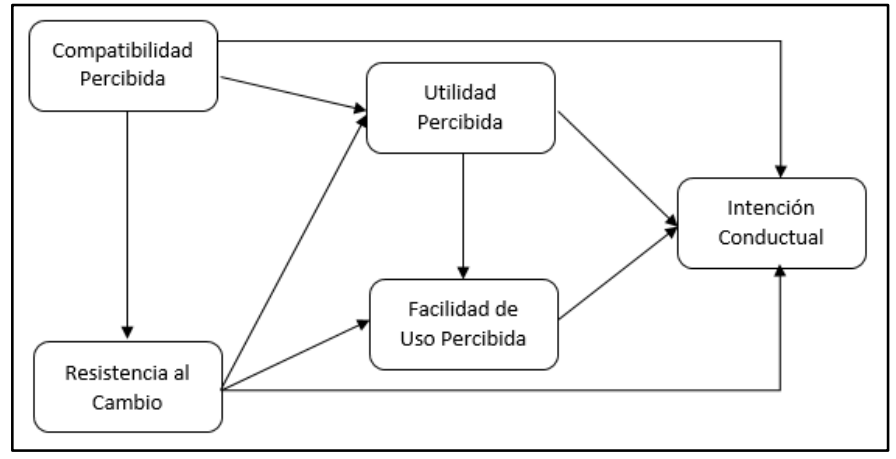

Figura. 2. Esquema del modelo de investigación.

Al ser un constructo poco explorado, que consideramos puede tener una influencia significativa en la aceptación de las tecnologías móviles por parte de los docentes de primaria, planteamos el estudio de sus relaciones con los tres constructos incorporados del modelo TAM. Además, teniendo en cuenta la estrecha relación entre este constructo y la compatibilidad percibida, también planteamos como hipótesis la relación positiva 
entre ambas (Figura 2). Las hipótesis planteadas para este constructo son, por tanto, las siguientes:

- H6: La resistencia al cambio está positivamente relacionada con la intención de uso de tecnologías móviles de los estudiantes de magisterio en su futura práctica docente.

- H7: La resistencia al cambio está positivamente relacionada con la utilidad percibida por los estudiantes en el uso de tecnologías móviles en su futura práctica docente.

- H8: La resistencia al cambio está positivamente relacionada con la facilidad de uso de tecnologías móviles en la enseñanza percibido por los estudiantes de magisterio.

- H9: La resistencia al cambio está negativamente relacionada con la compatibilidad percibida.

\section{Variables}

Para el presente trabajo de investigación se plantean las siguientes variables:

- Exógenas: Utilidad percibida, facilidad de uso percibida, compatibilidad y resistencia al cambio.

- Endógenas: Intención conductual.

- Otras variables explicativas: Edad, género, curso y centro.

\section{Población y Muestra}

La población de este estudio está constituida por los estudiantes matriculados en el Grado de Maestro de Educación Primaria de la Universidad de Salamanca en sus sedes de Salamanca $(\mathrm{N}=480)$, Zamora $(\mathrm{N}=320)$ y Ávila $(\mathrm{N}=234)$ durante el curso 14/15.

En total participaron 678 estudiantes: el 48,2\% (327) de la Facultad de Educación de Salamanca, el 26,1\% (177) de la Escuela de Educación y Turismo de Ávila y el 25,7\% (174) de la Escuela Universitaria de Magisterio de Zamora.

En cuanto a la distribución por género, el $65,2 \%$ de los participantes son mujeres, mientras que el 34,8\% son hombres. (Figura 4). El 51,3\% de los encuestados tienen una edad situada entre 19 y 21 años, siendo la media de edad 21,09 años.

Por último, la distribución por cursos de los alumnos es $29,8 \%$ de estudiantes de primero, $27,9 \%$ de estudiantes de segundo curso, $19,5 \%$ de tercero y $22,9 \%$ de cuarto.

\section{Instrumento}

Para realizar el proceso de recogida de datos se confeccionó un instrumento, siguiendo la propuesta de Davis, dividido en dos secciones. La primera de ellas está dedicada a recoger los datos de identificación de los estudiantes (edad, género y curso). La segunda está compuesta por 16 ítems formulados en escala de Likert de siete intervalos (0-6) que recogen el resto de variables. Los ítems se presentan mezclados al azar y sin agrupar ni etiquetar para evitar inflar la fiabilidad de manera artificial (Goodhue y Loiacono, 2002), 
aunque existe disparidad de opiniones al respecto en la comunidad científica (Davis y Venkatesh, 1996). Los ítems utilizados en este estudio son una adaptación de una propuesta realizada con anterioridad (Sánchez-Prieto, Olmos-Migueláñez y GarcíaPeñalvo, 2016), que cuentan con una validación de contenido mediante juicio de expertos. En la presente investigación pretendemos profundizar en el proceso de validación de dichos ítems.

En la propuesta original, los ítems referidos a la facilidad de uso y la utilidad percibida fueron elaborados a partir de la aportación de Davis (1989):

- Utilidad percibida (PU):

- PU_01: El uso de tecnologías móviles puede mejorar el desempeño de la labor docente.

- PU_02: El uso de tecnologías móviles puede hacer que sea más efectivo el desempeño de la labor docente

- PU_03: El uso de dispositivos móviles puede hacer más fácil realizar tareas docentes.

- PU_04: En general considero que los dispositivos móviles pueden ser útiles en la enseñanza.

\section{- Facilidad de uso percibida (PEU):}

- PEU_01: Aprender a utilizar dispositivos móviles en clase sería fácil para mí.

○ PEU_02: Encuentro fácil la interacción con dispositivos móviles.

○ PEU_03: Encuentro flexible la interacción con dispositivos móviles.

○ PEU_04: En general considero que los dispositivos móviles son fáciles de utilizar.

Para la intención conductual de uso se elaboraron dos ítems basados en los propuestos en el TAM 3 (Venkatesh y Bala, 2008):

- Intención conductual de uso (BI):

○ BI_01: Pretendo utilizar las tecnologías móviles en mi futura labor docente

○ BI_02: Predigo que utilizaría las tecnologías móviles en mi futura labor docente.

Para el constructo de compatibilidad percibida nos hemos basado en la propuesta de Moore y Benbasat (1991):

\section{- Compatibilidad Percibida (PC):}

- PC_01: Utilizar tecnologías móviles en la enseñanza sería compatible con mi estilo docente. 
- PC_02: Utilizar tecnologías móviles para enseñar sería coherente con mi forma de pensar.

- PC_03: Utilizar tecnologías móviles en la docencia encajaría con mi estilo de vida.

Por último, para la resistencia al cambio, se han partido de las propuestas de Bhattacherjee y Hikmet (2007) y Guo et al. (2013), quienes elaboran unos indicadores formulados de manera inversa.

\section{- Resistencia al cambio (RC):}

- RC_01: No me gustaría que las tecnologías móviles cambien la manera en que se desarrolla la función docente.

- RC_02: No quiero que las tecnologías móviles cambien la interacción profesor-alumno.

- RC_03: Asumir los cambios en la metodología docente que suponen las tecnologías móviles me resultaría fácil.

Para evaluar la consistencia interna del instrumento se ha utilizado el coeficiente $\alpha$ de Cronbach, cuyo resultado indica una alta consistencia interna $(\alpha=.862)$. A continuación presentamos una tabla comparativa (Tabla 4) que recoge los resultados de los índices de consistencia interna de los constructos obtenidos en nuestra investigación y los de los instrumentos a partir de los cuales fueron elaborados.

Tabla. 4. Comparativa entre los índices de consistencia interna.

\begin{tabular}{|c|c|c|c|}
\hline Constructo & Propuesta Original & Elaboración Propia & Tipo de Índice \\
\hline Utilidad percibida & .98 & .78 & Alfa de Cronbach \\
\hline $\begin{array}{c}\text { Facilidad de uso } \\
\text { percibida }\end{array}$ & .94 & .75 & Alfa de Cronbach \\
\hline $\begin{array}{c}\text { Compatibilidad } \\
\text { percibida }\end{array}$ & $.88-.83$ & .80 & Alfa de Cronbach \\
\hline Resistencia al cambio & $.94-.92$ & .68 & IFC \\
\hline Intención de uso & .90 & .83 & IFC \\
\hline
\end{tabular}

\section{Resultados}

En el presente apartado presentamos los resultados obtenidos en la investigación. La sección consta de dos partes. La primera de ellas está destinada a exponer el resultado del análisis descriptivo y contraste de las hipótesis relacionadas con la influencia de los factores demográficos: Curso, género y centro de pertenencia.

En la segunda parte recogeremos los resultados del análisis factorial realizado con la intención de validar el instrumento.

\section{Análisis Descriptivo y Contraste de Hipótesis}

Con la intención de realizar la evaluación de la intención conductual de uso de tecnologías móviles en la futura práctica docente de los alumnos del Grado de Maestro de Educación Primaria, a continuación presentamos los resultados obtenidos del análisis 
descriptivo realizado, organizados por constructos (Tabla 5). Los ítems RC_01 y RC_02 correspondientes a la resistencia al cambio se recodificaron al tratarse de ítems negativos.

Los resultados obtenidos muestran una actitud positiva por parte de los estudiantes hacia la inclusión de los dispositivos móviles durante el futuro ejercicio de su posición, habiendo obtenido puntuaciones por encima del 4, sobre un máximo de 6 en 12 de los 16 ítems. De los 4 restantes, 2 de ellos pertenecientes al constructo de compatibilidad percibida, PC_02 y PC_03 presentan puntuaciones por encima del tres. Los 2 ítems que quedan, RC_01 y RC_02, tienen puntuaciones por debajo del punto medio de la escala. Esto indica que los factores resistencia al cambio y compatibilidad percibida son susceptibles de intervención.

Tabla. 5. Estadísticos descriptivos de los ítems del modelo propuesto.

\begin{tabular}{|c|c|c|c|c|c|c|c|c|c|c|}
\hline & \multirow[b]{2}{*}{ Media } & \multirow[b]{2}{*}{ Desv.Tip. } & \multicolumn{7}{|c|}{ \% Válido } & \multirow[b]{2}{*}{$\mathbf{N}$} \\
\hline & & & 0 & 1 & 2 & 3 & 4 & 5 & 6 & \\
\hline PEU_04 & 4.55 & 1.222 & .6 & 1.9 & 4.3 & 9.2 & 25.7 & 35.7 & 22.6 & 676 \\
\hline PEU_01 & 4.49 & 1.282 & .6 & 2.8 & 3.2 & 13.9 & 22.9 & 33.3 & 23.3 & 678 \\
\hline PEU_02 & 4.45 & 1.213 & 1.2 & 1.2 & 3.6 & 12.0 & 27.8 & 35.2 & 18.9 & 665 \\
\hline PU_04 & 4.32 & 1.285 & 1.3 & 3.3 & 4.3 & 11.7 & 25.3 & 40.4 & 13.6 & 668 \\
\hline PU_01 & 4.20 & 1.328 & 1.2 & 2.8 & 7.2 & 14.0 & 28.5 & 30.7 & 15.6 & 678 \\
\hline RC_03 & 4.18 & 1.314 & 1.0 & 3.9 & 6.0 & 13.5 & 30.4 & 31.4 & 13.8 & 672 \\
\hline BI_01 & 4.15 & 1.450 & 2.1 & 3.9 & 6.1 & 17.8 & 23.9 & 27.7 & 18.7 & 675 \\
\hline PU_03 & 4.10 & 1.301 & 1.2 & 3.0 & 7.2 & 17.0 & 27.5 & 32.9 & 11.3 & 666 \\
\hline PC_01 & 4.08 & 1.357 & 1.9 & 3.8 & 4.9 & 19.7 & 24.0 & 34.3 & 11.4 & 676 \\
\hline PU_02 & 4.08 & 1.272 & 1.6 & 2.8 & 5.2 & 18.4 & 30.0 & 32.1 & 9.7 & 669 \\
\hline PEU_03 & 4.06 & 1.221 & .6 & 2.8 & 3.2 & 13.9 & 22.9 & 33.3 & 23.3 & 659 \\
\hline BI_02 & 4.03 & 1.418 & 2.1 & 4.7 & 6.3 & 18.1 & 25.2 & 31.0 & 12.7 & 664 \\
\hline PC_03 & 3.91 & 1.392 & 1.6 & 5.1 & 7.9 & 20.5 & 26.6 & 27.7 & 10.6 & 669 \\
\hline PC_02 & 3.87 & 1.397 & 2.5 & 4.5 & 7.6 & 21.3 & 25.2 & 30.4 & 2.5 & 670 \\
\hline RC_01 & 2.40 & 1.698 & 15.9 & 17.7 & 18.8 & 23.8 & 10.0 & 8.6 & 15.9 & 671 \\
\hline RC_02 & 2.01 & 1.736 & 24.1 & 21.4 & 18.0 & 18.1 & 7.5 & 5.7 & 5.2 & 668 \\
\hline
\end{tabular}

a Dimensiones organizadas por el valor de la media.

Una vez conocidos los descriptivos generales, interesa comprobar si hay diferencias significativas en función del curso género, curso y centro de pertenencia de los estudiantes.

Comenzamos por la variable curso. Para ello comenzamos elaborando un estudio de las variables diferenciando en función del curso (Tabla 6). A simple vista no se aprecian grandes diferencias, aunque sí existen algunas discrepancias, por lo que decidimos llevar a cabo un contraste hipótesis para determinar si se trata o no de diferencias significativas.

Tabla. 6. Descriptiva del modelo en función del curso.

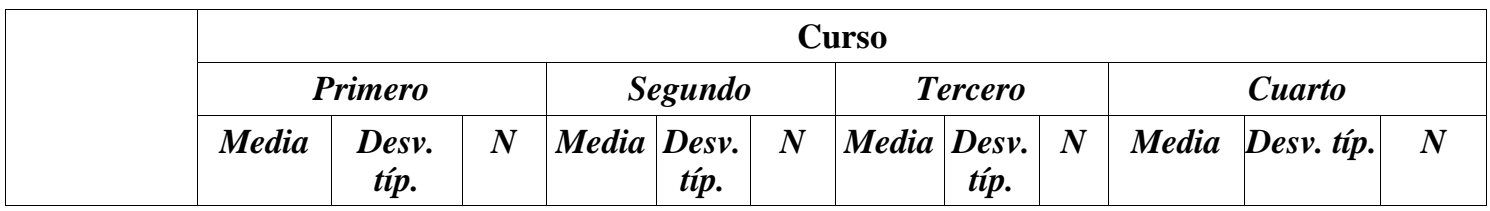




\begin{tabular}{|c|c|c|c|c|c|c|c|c|c|c|c|c|}
\hline BI_01 & 4.15 & 1.399 & 200 & 3.95 & 1.534 & 189 & 4.39 & 1.259 & 132 & 4.19 & 1.539 & 154 \\
\hline BI_02 & 4.05 & 1.431 & 198 & 3.99 & 1.365 & 184 & 4.19 & 1.278 & 128 & 3.92 & 1.566 & 154 \\
\hline PC_01 & 4.09 & 1.402 & 202 & 4.13 & 1.283 & 188 & 4.11 & 1.225 & 132 & 3.99 & 1.493 & 154 \\
\hline PC_02 & 3.92 & 1.344 & 199 & 3.87 & 1.446 & 189 & 3.83 & 1.301 & 130 & 3.83 & 1.491 & 152 \\
\hline PC_03 & 4.01 & 1.391 & 201 & 3.76 & 1.375 & 186 & 4.12 & 1.177 & 131 & 3.75 & 1.553 & 151 \\
\hline PEU_01 & 4.53 & 1.316 & 202 & 4.39 & 1.327 & 189 & 4.69 & 1.027 & 132 & 4.38 & 1.364 & 155 \\
\hline PEU_02 & 4.46 & 1.246 & 199 & 4.39 & 1.222 & 183 & 4.63 & 1.035 & 130 & 4.37 & 1.292 & 153 \\
\hline PEU_03 & 4.11 & 1.120 & 195 & 3.93 & 1.286 & 182 & 4.19 & 1.050 & 130 & 4.01 & 1.386 & 152 \\
\hline PEU_04 & 4.60 & 1.231 & 202 & 4.51 & 1.290 & 188 & 4.70 & .990 & 131 & 4.41 & 1.293 & 155 \\
\hline PU_01 & 4.01 & 1.369 & 202 & 4.17 & 1.249 & 189 & 4.45 & 1.231 & 132 & 4.28 & 1.417 & 155 \\
\hline PU_02 & 4.04 & 1.235 & 200 & 3.92 & 1.304 & 185 & 4.31 & 1.167 & 130 & 4.11 & 1.346 & 154 \\
\hline PU_03 & 4.05 & 1.324 & 201 & 3.98 & 1.351 & 184 & 4.32 & 1.188 & 130 & 4.13 & 1.289 & 151 \\
\hline PU_04 & 4.27 & 1.365 & 199 & 4.27 & 1.226 & 185 & 4.52 & 1.176 & 130 & 4.28 & 1.331 & 154 \\
\hline RC_01 & 2.48 & 1.588 & 200 & 2.51 & 1.909 & 185 & 2.15 & 1.489 & 131 & 2.39 & 1.726 & 155 \\
\hline RC_02 & 2.04 & 1.586 & 200 & 2.11 & 1.884 & 183 & 1.88 & 1.664 & 131 & 1.99 & 1.806 & 154 \\
\hline RC_03 & 4.24 & 1.296 & 200 & 4.10 & 1.275 & 187 & 4.36 & 1.085 & 132 & 4.05 & 1.536 & 153 \\
\hline
\end{tabular}

a Las dimensiones se presentan organizadas alfabéticamente.

Aplicamos las pruebas de normalidad de Kolmogorov-Smirnov y Shapiro Wilk (Tabla 7) con el objetivo de seleccionar el método más adecuado para el contraste de hipótesis. Los resultados implican el rechazo a la hipótesis de normalidad (n.s. .05), por lo que se empleará estadística no paramétrica.

Tabla. 7. Test de normalidad de Kolmogorov-Smirnov y Shapiro-Wilk.

\begin{tabular}{|c|c|c|c|c|c|c|}
\hline & \multicolumn{3}{|c|}{ Kolmogorov-Smirnov } & \multicolumn{3}{c|}{ Shapiro-Wilk } \\
\cline { 2 - 7 } & Estadístico & $\boldsymbol{g} \boldsymbol{l}$ & Sig. & Estadístico & $\boldsymbol{g l}$ & Sig. \\
\hline BI_01 & .190 & 580 & .000 & .900 & 580 & .000 \\
\hline BI_02 & .196 & 580 & .000 & .904 & 580 & .000 \\
\hline PC_01 & .213 & 580 & .000 & .893 & 580 & .000 \\
\hline PC_02 & .180 & 580 & .000 & .913 & 580 & .000 \\
\hline PC_03 & .173 & 580 & .000 & .920 & 580 & .000 \\
\hline PEU_01 & .226 & 580 & .000 & .876 & 580 & .000 \\
\hline PEU_02 & .211 & 580 & .000 & .883 & 580 & .000 \\
\hline PEU_03 & .207 & 580 & .000 & .892 & 580 & .000 \\
\hline PEU_04 & .224 & 580 & .000 & .873 & 580 & .000 \\
\hline PU_01 & .195 & 580 & .000 & .897 & 580 & .000 \\
\hline PU_02 & .201 & 580 & .000 & .897 & 580 & .000 \\
\hline PU_03 & .200 & 580 & .000 & .905 & 580 & .000 \\
\hline PU_04 & .241 & 580 & .000 & .864 & 580 & .000 \\
\hline RC_01 & .130 & 580 & .000 & .930 & 580 & .000 \\
\hline RC_02 & .182 & 580 & .000 & .896 & 580 & .000 \\
\hline RC_03 & .208 & 580 & .000 & .892 & 580 & .000 \\
\hline
\end{tabular}

aCorrección de la significación de Liliefors.

También realizamos el cálculo del coeficiente de curtosis y asimetría (n.s. .05) para conocer grado de concentración que presentan los valores alrededor de la zona central de la distribución (Tabla 8). 
Tabla. 8. Coeficiente de Curtosis de los ítems.

\begin{tabular}{|c|c|c|c|c|}
\hline & $\begin{array}{c}\text { Coef. de } \\
\text { Curtosis }\end{array}$ & $\begin{array}{c}\text { Error est. } \\
\text { de Curt. }\end{array}$ & $\begin{array}{c}\text { Coef. de } \\
\text { Asimetría }\end{array}$ & $\begin{array}{c}\text { Error est. } \\
\text { de Asim. }\end{array}$ \\
\hline BI_01 & .088 & .189 & -.733 & .094 \\
\hline BI_02 & .168 & .190 & -.763 & .095 \\
\hline PC_01 & .374 & .188 & -.832 & .094 \\
\hline PC_02 & .134 & .189 & -.708 & .094 \\
\hline PC_03 & -.109 & .189 & -.596 & .094 \\
\hline PEU_01 & .560 & .188 & -.884 & .094 \\
\hline PEU_02 & 1.336 & .190 & -.975 & .095 \\
\hline PEU_03 & .693 & .191 & -.826 & .095 \\
\hline PEU_04 & .839 & .188 & -.988 & .094 \\
\hline PU_01 & .174 & .188 & -.753 & .094 \\
\hline PU_02 & .677 & .189 & -.826 & .094 \\
\hline PU_03 & .217 & .190 & -.737 & .095 \\
\hline PU_04 & 1.061 & .190 & -1.107 & .095 \\
\hline RC_01 & -.687 & .189 & .330 & .094 \\
\hline RC_02 & -.422 & .190 & .651 & .095 \\
\hline RC_03 & .441 & .189 & -.811 & .094 \\
\hline
\end{tabular}

Como puede verse en la tabla 8, de los 16 ítems que componen el instrumento, 8 presentan una distribución leptocúrtica PC_01, PEU_01, PEU_02, PEU_03, PEU_04, PU_02, PU_04 y RC_03. De los restantes, 6 tienen una distribución mesocurtíca: BI_01, BI_02, PC_02, PC_03, PU_01 y PU_03. Los dos ítems restantes, RC_01 y RC02, tienen una distribución platocúrtica. En ninguno de los casos se han encontrado puntuaciones de curtosis extremas, permitiéndose por tanto el análisis factorial que presentaremos en el siguiente apartado.

En cuanto a la asimetría, todos los ítems tienen una distribución asimétrica negativa, salvo los ítems RC_01 y RC_02 que presentan una asimetría positiva.

El estadístico seleccionado para el contraste de hipótesis es test de Kruskal Wallis (Tabla 9). Los resultados muestran que solamente existen diferencias significativas en 2 de los 16 ítems, más concretamente en los ítems PU_01 y PU_02 (n.s. .05) en los que los estudiantes de tercero presentan las puntuaciones medias más altas y los de primero y segundo las más bajas respectivamente.

Tabla. 9. Resultados Kruskal Wallis para la variable curso.

\begin{tabular}{|c|c|c|c|}
\hline & Chi-Cuadrado & Df & Sig. Asymp. \\
\hline BI_01 & 6.253 & 3 & .100 \\
\hline BI_02 & 1.771 & 3 & .621 \\
\hline PC_01 & .370 & 3 & .946 \\
\hline PC_02 & .420 & 3 & .936 \\
\hline PC_03 & 6.109 & 3 & .106 \\
\hline PEU_01 & 3.378 & 3 & .337 \\
\hline
\end{tabular}




\begin{tabular}{|c|c|c|c|}
\hline PEU_02 & 2.511 & 3 & .473 \\
\hline PEU_03 & 2.570 & 3 & .463 \\
\hline PEU_04 & 2.864 & 3 & .413 \\
\hline PU_01 & 9.277 & 3 & .026 \\
\hline PU_02 & 8.129 & 3 & .043 \\
\hline PU_03 & 5.724 & 3 & .126 \\
\hline PU_04 & 3.641 & 3 & .303 \\
\hline RC_01 & 3.378 & 3 & .337 \\
\hline RC_02 & 1.251 & 3 & .741 \\
\hline RC_03 & 3.353 & 3 & .340 \\
\hline
\end{tabular}

El segundo factor que se desea considerar es el género. Para ello, se sigue el mismo procedimiento para verificar si existen diferencias significativas en las medias (n.s. .05): primero se lleva a cabo un estudio descriptivo diferenciando en función de la variable género (Tabla 10) y, por último, dadas las numerosas diferencias observadas en las medias, se calcula en índice U de Mann-Whitney (Tabla 11), más apropiado para 2 muestras independientes.

Tabla. 10. Descriptiva del modelo en función del género.

\begin{tabular}{|c|c|c|c|c|c|c|}
\hline \multirow{2}{*}{} & \multicolumn{5}{|c|}{ Género de los estudiantes } \\
\cline { 2 - 7 } & \multicolumn{7}{|c|}{ Mujer } & \multicolumn{3}{c|}{ Hombre } \\
\cline { 2 - 7 } & Media & Desv. típ. & $\boldsymbol{N}$ & Media & Desv. típ. & $\boldsymbol{N}$ \\
\hline BI_01 & 4.10 & 1.486 & 438 & 4.28 & 1.361 & 234 \\
\hline BI_02 & 3.98 & 1.411 & 433 & 4.14 & 1.427 & 228 \\
\hline PC_01 & 4.01 & 1.357 & 440 & 4.23 & 1.351 & 233 \\
\hline PC_02 & 3.78 & 1.389 & 432 & 4.06 & 1.378 & 235 \\
\hline PC_03 & 3.83 & 1.371 & 435 & 4.07 & 1.417 & 231 \\
\hline PEU_01 & 4.39 & 1.337 & 440 & 4.69 & 1.148 & 235 \\
\hline PEU_02 & 4.40 & 1.247 & 434 & 4.59 & 1.101 & 228 \\
\hline PEU_03 & 4.00 & 1.246 & 428 & 4.19 & 1.155 & 228 \\
\hline PEU_04 & 4.52 & 1.241 & 440 & 4.62 & 1.180 & 233 \\
\hline PU_01 & 4.15 & 1.354 & 440 & 4.34 & 1.258 & 235 \\
\hline PU_02 & 3.98 & 1.259 & 432 & 4.28 & 1.252 & 234 \\
\hline PU_03 & 4.03 & 1.325 & 431 & 4.26 & 1.242 & 232 \\
\hline PU_04 & 4.26 & 1.307 & 432 & 4.44 & 1.235 & 234 \\
\hline RC_01 & 2.28 & 1.662 & 437 & 2.63 & 1.749 & 231 \\
\hline RC_02 & 1.92 & 1.705 & 437 & 2.19 & 1.788 & 228 \\
\hline RC_03 & 4.16 & 1.330 & 437 & 4.23 & 1.286 & 233 \\
\hline
\end{tabular}

Tabla. 11. Resultados U de Mann-Whitney para la variable género.

\begin{tabular}{|c|c|c|c|c|}
\hline & U de Mann-Whitney & W de Wilcoxon & Z & Sig. asintót. (bilateral) \\
\hline BI_01 & 48154.000 & 144295.000 & -1.322 & .186 \\
\hline
\end{tabular}

Resistencia al Cambio y Compatibilidad con las Tecnologías Móviles. Un Estudio Sobre la Intención de Uso Entre los Futuros Docentes. José Carlos Sánchez-Prieto, Susana Olmos-Migueláñez, Francisco J.

García-Peñalvo.

Página 31 de 31 


\begin{tabular}{|c|c|c|c|c|}
\hline BI_02 & 45640.000 & 139601.000 & -1.640 & .101 \\
\hline PC_01 & 46294.000 & 143314.000 & -2.139 & .032 \\
\hline PC_02 & 45008.500 & 138536.500 & -2.490 & .013 \\
\hline PC_03 & 44686.500 & 139516.500 & -2.413 & .016 \\
\hline PEU_01 & 45605.000 & 142625.000 & -2.611 & .009 \\
\hline PEU_02 & 45713.500 & 140108.500 & -1.672 & .094 \\
\hline PEU_03 & 44369.000 & 136175.000 & -1.990 & .047 \\
\hline PEU_04 & 49210.500 & 146230.500 & -.888 & .375 \\
\hline PU_01 & 47886.000 & 144906.000 & -1.630 & .103 \\
\hline PU_02 & 43153.500 & 136681.500 & -3.230 & .001 \\
\hline PU_03 & 45251.000 & 138347.000 & -2.084 & .037 \\
\hline PU_04 & 46285.500 & 139813.500 & -1.880 & .060 \\
\hline RC_01 & 45006.500 & 140709.500 & -2.342 & .019 \\
\hline RC_02 & 45331.500 & 141034.500 & -1.944 & .052 \\
\hline RC_03 & 49378.500 & 145081.500 & -.664 & .507 \\
\hline
\end{tabular}

Como se puede comprobar en la tabla 11, se han encontrado diferencias significativas en 9 de los 16 ítems propuestos. Esta diferencia es especialmente relevante en el caso de la compatibilidad percibida, ya que en los tres ítems de este constructo la diferencia es significativa. En el caso de la resistencia al cambio, se encuentran diferencias en 2 de sus 3 indicadores: RC_02 y RC_03. El resto de ítems son: PEU_01, PEU_03, PU_02 y PU03.

En todos los casos los hombres obtienen puntuaciones medias superiores a las de las mujeres, lo que lleva a pensar que tienen una mejor disposición al uso de estas tecnologías para la docencia.

Por último, en esta fase de contraste de hipótesis queremos comprobar si existen diferencias en función del centro de pertenencia de los estudiantes, al igual que con las variables anteriores comenzamos realizando un análisis descriptivo (Tabla 12), tras lo cual aplicamos el test de Kruskal Wallis al tratarse de más de 2 muestras independientes (Tabla 13).

Tabla. 12. Descriptiva del modelo en función del centro.

\begin{tabular}{|c|c|c|c|c|c|c|c|c|c|}
\hline & \multicolumn{9}{|c|}{ Centro } \\
\cline { 2 - 11 } & \multicolumn{3}{|c|}{ Salamanca } & \multicolumn{3}{c|}{ Zamora } & \multicolumn{3}{c|}{ Ávila } \\
\cline { 2 - 11 } & Media & Desv. típ. & $\boldsymbol{N}$ & Media & Desv. típ. & $\boldsymbol{N}$ & Media & Desv. típ. & $\boldsymbol{N}$ \\
\hline BI_01 & 4.16 & 1.418 & 326 & 4.11 & 1.511 & 176 & 4.18 & 1.454 & 173 \\
\hline BI_02 & 4.10 & 1.383 & 323 & 3.97 & 1.477 & 171 & 3.96 & 1.424 & 170 \\
\hline PC_01 & 4.15 & 1.329 & 326 & 3.93 & 1.430 & 177 & 4.12 & 1.326 & 173 \\
\hline PC_02 & 3.92 & 1.393 & 323 & 3.79 & 1.415 & 175 & 3.85 & 1.389 & 172 \\
\hline PC_03 & 3.85 & 1.364 & 323 & 3.83 & 1.510 & 175 & 4.09 & 1.307 & 171 \\
\hline PEU_01 & 4.44 & 1.271 & 327 & 4.31 & 1.336 & 177 & 4.75 & 1.213 & 174 \\
\hline PEU_02 & 4.43 & 1.231 & 321 & 4.35 & 1.162 & 172 & 4.61 & 1.221 & 172 \\
\hline PEU_03 & 4.05 & 1.218 & 314 & 4.03 & 1.176 & 173 & 4.08 & 1.277 & 172 \\
\hline
\end{tabular}

Resistencia al Cambio y Compatibilidad con las Tecnologías Móviles. Un Estudio Sobre la Intención de Uso Entre los Futuros Docentes. José Carlos Sánchez-Prieto, Susana Olmos-Migueláñez, Francisco J. 


\begin{tabular}{|c|c|c|c|c|c|c|c|c|c|}
\hline PEU_04 & 4.55 & 1.254 & 326 & 4.42 & 1.228 & 177 & 4.69 & 1.144 & 173 \\
\hline PU_01 & 4.20 & 1.305 & 327 & 4.10 & 1.349 & 177 & 4.32 & 1.347 & 174 \\
\hline PU_02 & 4.07 & 1.193 & 322 & 3.95 & 1.338 & 175 & 4.22 & 1.340 & 172 \\
\hline PU_03 & 4.12 & 1.243 & 322 & 3.89 & 1.414 & 174 & 4.29 & 1.261 & 170 \\
\hline PU_04 & 4.38 & 1.200 & 320 & 4.14 & 1.366 & 174 & 4.39 & 1.342 & 174 \\
\hline RC_01 & 2.53 & 1.707 & 323 & 2.23 & 1.604 & 175 & 2.35 & 1.764 & 173 \\
\hline RC_02 & 2.08 & 1.751 & 323 & 1.84 & 1.687 & 173 & 2.06 & 1.754 & 172 \\
\hline RC_03 & 4.15 & 1.259 & 323 & 3.99 & 1.324 & 175 & 4.41 & 1.377 & 174 \\
\hline
\end{tabular}

a Las dimensiones se presentan organizadas alfabéticamente.

Tabla. 13. Resultados Kruskal Wallis para la variable centro.

\begin{tabular}{|c|c|c|c|}
\hline & Chi-Cuadrado & df & Sig. Asymp. \\
\hline BI_01 & .112 & 2 & .946 \\
\hline BI_02 & 1.464 & 2 & .481 \\
\hline PC_01 & 2.762 & 2 & .251 \\
\hline PC_02 & 1.149 & 2 & .563 \\
\hline PC_03 & 3.854 & 2 & .146 \\
\hline PEU_01 & 12.713 & 2 & .002 \\
\hline PEU_02 & 5.775 & 2 & .056 \\
\hline PEU_03 & .468 & 2 & .791 \\
\hline PEU_04 & 4.720 & 2 & .094 \\
\hline PU_01 & 4.308 & 2 & .116 \\
\hline PU_02 & 5.035 & 2 & .081 \\
\hline PU_03 & 7.254 & 2 & .027 \\
\hline PU_04 & 5.145 & 2 & .076 \\
\hline RC_01 & 3.227 & 2 & .199 \\
\hline RC_02 & 2.375 & 2 & .305 \\
\hline RC_03 & 12.348 & 2 & .002 \\
\hline
\end{tabular}

Los resultados del contraste de hipótesis reflejado en la tabla 13 muestran que existen diferencias significativas en tres ítems: uno perteneciente a la resistencia al cambio (RC_03), otro a la utilidad percibida (PU_03), y otro a la facilidad de uso percibida (PEU_01). En los tres ítems los estudiantes de Escuela Universitaria de Magisterio de Zamora han obtenido las puntuaciones medias más bajas y los de la Escuela de Educación y Turismo de Ávila las más altas.

\section{Validación de la escala}

\section{Validez Convergente}

Para medir la validez convergente del instrumento seguimos la propuesta de Fornel y Larcker (1981) quienes establecen tres procedimientos:

- Fiabilidad de los ítems de cada medida.

Resistencia al Cambio y Compatibilidad con las Tecnologías Móviles. Un Estudio Sobre la Intención de Uso Entre los Futuros Docentes. José Carlos Sánchez-Prieto, Susana Olmos-Migueláñez, Francisco J.

García-Peñalvo.

Página 31 de 31 
- Fiabilidad compuesta de cada constructo

- Varianza media extraída.

Para calcular la fiabilidad de los ítems de cada medida realizamos un análisis factorial exploratorio para comprobar la carga de los factores en cada uno de los constructos planteados.

Como método de extracción de factores principales empleamos el de ejes principales, considerado el más apropiado para los casos en los que la distribución muestral no cumple el supuesto de normalidad (Lloret-Segura, Ferreres-Traver, Hernández-Baeza y Tomás-Marco, 2014). Como método de rotación de los factores empleamos Oblimin (Clarkson y Jenrich, 1988), el método de rotación oblicua más habitual, al suponerse que existe relación entre los distintos constructos del modelo (Tabla 14).

Tabla. 14. Resultados análisis factorial.

\begin{tabular}{|c|c|c|c|c|c|}
\hline & \multicolumn{5}{|c|}{ Factor } \\
\cline { 2 - 6 } & $\boldsymbol{B I}$ & $\boldsymbol{P U}$ & $\boldsymbol{P E U}$ & $\boldsymbol{P C}$ & $\boldsymbol{R} \boldsymbol{C}$ \\
\hline BI_01 & $\mathbf{. 8 5 6}$ & .697 & .243 & -.524 & .089 \\
\hline BI_02 & $\mathbf{. 8 3 8}$ & .730 & .333 & -.621 & .191 \\
\hline PU_02 & .619 & $\mathbf{. 7 8 8}$ & .286 & -.514 & .140 \\
\hline PU_03 & .682 & $\mathbf{. 7 1 1}$ & .344 & -.481 & .145 \\
\hline PU_04 & .655 & $\mathbf{. 7 3 4}$ & .265 & -.568 & .179 \\
\hline PEU_01 & .376 & .411 & $\mathbf{. 6 7 9}$ & -.467 & .111 \\
\hline PEU_02 & .299 & .333 &. $\mathbf{7 3 2}$ & -.274 & .102 \\
\hline PEU_04 & .104 & .170 &. $\mathbf{7 8 0}$ & -.211 & .094 \\
\hline PC_01 & .642 & .684 & .390 & -.954 & .303 \\
\hline PC_02 & .668 & .681 & .411 & -.687 & .287 \\
\hline RC_01 & .155 & .168 & .077 & -.259 &. $\mathbf{7 3 5}$ \\
\hline RC_02 & .028 & .087 & .099 & -.149 & $\mathbf{. 7 0 7}$ \\
\hline
\end{tabular}

Para confirmar la validez de cada ítem en una dimensión se recomienda una puntuación absoluta superior a .70, siendo aceptable una puntuación absoluta por encima de $.50 \mathrm{y}$ entendiéndose como una carga marginal las puntuaciones absolutas por encima de .30 , por debajo de esta cifra se interpreta que no tienen un peso significativo.

Como se puede comprobar en la tabla, los ítems planteados presentan las puntuaciones más altas en las dimensiones para las que fueron planteados, con puntuaciones en la mayoría de los casos por encima del límite de .70. Durante el proceso fue necesario suprimir algunos de los ítems al presentar problemas en el análisis factorial.

Para el segundo índice, que mide la fiabilidad compuesta de cada constructo, empleamos el coeficiente alfa de Cronbach (Tabla 15).

Tabla. 15. Resultados fiabilidad compuesta.

Resistencia al Cambio y Compatibilidad con las Tecnologías Móviles. Un Estudio Sobre la Intención de Uso Entre los Futuros Docentes. José Carlos Sánchez-Prieto, Susana Olmos-Migueláñez, Francisco J.

García-Peñalvo. 


\begin{tabular}{|l|l|}
\hline Constructo & Puntuación \\
\hline Intención Conductual (BI) & .830 \\
\hline Utilidad Percibida (PU) & .782 \\
\hline Facilidad de Uso Percibida (PEU) & .755 \\
\hline Compatibilidad Percibida (PC) & .801 \\
\hline Resistencia al Cambio (RC) & .660 \\
\hline
\end{tabular}

En este caso, se obtuvieron puntuaciones superiores a .70, el valor recomendado, en todos los constructos, salvo en el de la resistencia al cambio, que, aunque presenta una puntuación inferior, tiene un valor por encima del .65, por lo que no consideramos necesario eliminarla del modelo.

Por último, hemos calculado también la varianza media extraída (VME) (Tabla 16).

Tabla. 16. Resultados VME.

\begin{tabular}{|l|l|}
\hline Constructo & Puntuación \\
\hline Intención Conductual (BI) & .717 \\
\hline Utilidad Percibida (PU) & .554 \\
\hline Facilidad de Uso Percibida (PEU) & .730 \\
\hline Compatibilidad Percibida (PC) & .690 \\
\hline Resistencia al Cambio (RC) & .517 \\
\hline
\end{tabular}

La validez convergente se confirma si se obtienen puntuaciones por encima de .50 en cada constructo, como es el caso en nuestra propuesta.

\section{Validez Discriminante}

Podemos considerar que existe validez discriminante cuando la varianza entre los constructos del modelo es menor que la varianza que cada constructo comparte con sus indicadores (Fornell, Tellis y Zinkhan, 1982).

Para comprobar esta validez, se compara la raíz cuadrada de la VME con la correlación entre cada uno de los constructos. Si la raíz cuadrada de la varianza media extraída es mayor que la correlación entre los constructos, se demuestra que se cumple el principio de la validez discriminante. En la tabla 17 podemos observar las correlaciones entre los constructos de nuestro modelo y en la diagonal entre paréntesis el valor de la raíz cuadrada de la VME. Como podemos observar, todos los constructos tienen un valor de VME por encima del valor de correlación con los otros constructos, salvo en el caso de la utilidad percibida, que tiene un valor de correlación con la intención conductual tres centésimas superior a la raíz cuadrada de su VME. Al tratarse de 2 constructos fuertemente relacionados, consideramos mantenerlos en el modelo.

Tabla. 17. Resultados validez discriminante.

\begin{tabular}{|l|l|l|l|l|l|}
\hline Factor & BI & PEU & RC & PC & PU \\
\hline
\end{tabular}

Resistencia al Cambio y Compatibilidad con las Tecnologías Móviles. Un Estudio Sobre la Intención de Uso Entre los Futuros Docentes. José Carlos Sánchez-Prieto, Susana Olmos-Migueláñez, Francisco J.

García-Peñalvo.

Página 31 de 31 


\begin{tabular}{|c|c|c|c|c|c|}
\hline BI & $\mathbf{( . 8 4 6 )}$ & & & & \\
\hline PEU & .297 & $\mathbf{( . 7 3 0 )}$ & & & \\
\hline RC & .131 & .127 & $\mathbf{( . 7 1 9 )}$ & & \\
\hline PC & -.612 & -.368 & -.283 & $\mathbf{( . 8 3 0 )}$ & \\
\hline PU & .807 & .362 & .183 & -.646 & $\mathbf{( . 7 7 4 )}$ \\
\hline
\end{tabular}

\section{Ajuste del Modelo}

Para comprobar el ajuste entre los datos obtenidos y el modelo propuesto llevamos a cabo un análisis factorial confirmatorio (AFC), para lo que empleamos el programa AMOS 23.

En nuestro análisis seleccionamos los índices más habituales en este tipo de investigaciones: $\chi 2, \chi 2 /$ dif, RMSEA, CFI y TLI (Teo, 2012) (Tabla 17).

Tabla. 18. Resultados bondad de ajuste del modelo.

\begin{tabular}{|c|c|c|c|}
\hline $\begin{array}{c}\text { Índices de ajuste de } \\
\text { modelo }\end{array}$ & Valores & $\begin{array}{c}\text { Valores } \\
\text { recomendados }\end{array}$ & Referencias \\
\hline$\chi^{\mathbf{2}}$ & .000 & No significativo & $\begin{array}{c}\text { Klem (2000); Kline } \\
(2005) ; \text { McDonald y } \\
\text { Ho (2002) }\end{array}$ \\
\hline$\chi^{\mathbf{2} / \text { dif }}$ & 3.840 & $<3$ & Kline (2005) \\
\hline RMSEA & .065 & $<.08$ & McDonald y Ho (2002) \\
\hline CFI & .964 & $>.90$ & $\begin{array}{c}\text { Klem (2000); } \\
\text { McDonald y Ho (2002) }\end{array}$ \\
\hline TLI & .935 & $>.90$ & $\begin{array}{c}\text { Klem (2000); } \\
\text { McDonald y Ho (2002) }\end{array}$ \\
\hline
\end{tabular}

En la tabla 18 puede verse cómo el modelo presenta un nivel de ajuste aceptable en todos los índices salvo en los relacionados con $\chi 2$, satisfaciendo todos los valores recomendados. La validez del uso $\chi 2$ como indicador único de la bondad del ajuste del modelo es un tema muy discutido entre la comunidad de investigadores, dado que este índice presenta problemas de adecuación a la distribución de los datos, excesiva sensibilidad al tamaño muestral o al número de variables y se basa en una hipótesis excesivamente restrictiva (Hair, Black, Babin, Anderson y Tatham, 2006).

Con el objetivo de superar estas limitaciones, se recurre al resto de índices mostrados en la tabla, según los cuales el modelo presenta un nivel de ajuste correcto.

\section{Conclusiones}

Los resultados de esta investigación llevada a cabo con los estudiantes del Grado de Maestro de Educación Primaria de la Universidad de Salamanca muestran una intención conductual moderadamente propensa al uso de tecnologías móviles en su futura práctica docente, con resultados por encima del tres en todos los ítems salvo en el RC_01 y RC_02. Estos resultados indican que los factores compatibilidad y resistencia al cambio son susceptibles de mejora, a través de intervenciones educativas planificadas.

Resistencia al Cambio y Compatibilidad con las Tecnologías Móviles. Un Estudio Sobre la Intención de Uso Entre los Futuros Docentes. José Carlos Sánchez-Prieto, Susana Olmos-Migueláñez, Francisco J. 
Las puntuaciones medias obtenidas concuerdan con las de otros estudios sobre la aceptación tecnológica entre profesores en su periodo de formación universitaria (Teo, Lee, Chai y Wong, 2009; Teo y Noyes, 2011). Si comparamos las puntuaciones obtenidas con las de estudios realizados con profesores ya incorporados a su puesto de trabajo, podemos ver que estos presentan puntuaciones de corte similar (Fong et al., 2014; Wu y Liu, 2013), aunque existen estudios sobre el grado de aceptación de las nuevas tecnologías entre los docentes en las que obtienen puntuaciones inferiores en casos como los videojuegos educativos (Bourgonjon et al., 2013) o herramientas de LMS (Schoonenboom, 2014).

En cuanto al contraste de hipótesis relacionadas con los factores demográficos, observamos que existen diferencias significativas relacionadas con el centro de pertenencia de los estudiantes en tres de los 16 ítems. Esto indica que puede haber algunas diferencias en función de la metodología empleada en los centros, por lo que sería interesante profundizar en este campo en futuros análisis.

Para la variable curso, sólo se han encontrado diferencias significativas en dos de los ítems relacionados con la utilidad percibida. Esto puede ser indicativo de una falta de formación en el uso de estas herramientas, o de falta de participación de los estudiantes en experiencias de aprendizaje móvil (Lei, 2009).

Los resultados obtenidos implican, por tanto, la necesidad de incluir programas específicos de formación en mlearning, de cara a la mejora progresiva de la aceptación de estos dispositivos por parte de los alumnos a medida que avanza su proceso educativo.

Por último, llaman la atención las diferencias estadísticamente significativas encontradas en función de la variable género en más de la mitad de los ítems del instrumento, especialmente en el caso de la compatibilidad y la resistencia al cambio. La profundización en el estudio de la influencia de este factor en los constructos mencionados constituye un interesante campo de cara a futuras investigaciones.

El estudio de la influencia del género en el proceso de adopción supone un campo de interés en el que encontramos otros ejemplos de investigaciones que han encontrado diferencias entre hombres y mujeres (Padilla-Meléndez, del Aguila-Obra y GarridoMoreno, 2013), aunque esto no es siempre así (Ramírez-Correa, Rondán-Cataluña y Arenas-Gaitán, 2010), por lo que es necesario seguir profundizando.

En relación a la validación del instrumento, pese a algunas limitaciones, se han obtenido resultados satisfactorios tanto en las pruebas de validez convergente. Así mismo, a pesar de los problemas de validez discriminante detectados entre la intención conductual y la utilidad, el análisis factorial llevado a cabo refleja una aceptable bondad de ajuste del modelo.

Nuestra propuesta constituye por tanto un modelo validado para el estudio de la aceptación de tecnologías móviles que integra los constructos de compatibilidad y resistencia al cambio, dimensión que hasta ahora no habíamos encontrado incluida y validada en ningún estudio con este colectivo.

De cara a futuras investigaciones se plantea la posibilidad de realizar un análisis empleando modelos de ecuaciones estructurales SEM para comprobar las relaciones planteadas entre los constructos. Además, resultaría beneficioso refinar los ítems

Resistencia al Cambio y Compatibilidad con las Tecnologías Móviles. Un Estudio Sobre la Intención de Uso Entre los Futuros Docentes. José Carlos Sánchez-Prieto, Susana Olmos-Migueláñez, Francisco J. 
planteados para cada una de las dimensiones con el objetivo de reforzarlos y superar los problemas encontrados durante el proceso de validación.

\section{Agradecimientos}

Este trabajo de investigación ha sido realizado dentro del programa de Doctorado en Formación en la Sociedad del Conocimiento desarrollado en la Universidad de Salamanca.

Esta investigación ha sido financiada por la Universidad de Salamanca a través del "Programa III: Ayudas para contratos Predoctorales".

Presentación del artículo: 9 de marzo de 2016

Fecha de aprobación: 17 de mayo de 2016

Fecha de publicación: 30 de enero de 2017

Sánchez Prieto, J.C., Olmos Migueláñez, S., y García-Peñalvo, F.J. (2017). ¿Utilizarán los futuros docentes las tecnologías móviles? Validación de una propuesta de modelo TAM extendido. RED. Revista de Educación a Distancia, 52. Consultado el (dd/mm/aaaa) en http://www.um.es/ead/red/52

\section{Referencias}

Abbad, M. (2011). A conceptual model of factors affecting e-learning adoption Proceedings of the 2011 IEEE Global Engineering Education Conference (EDUCON) (pp. 1108-1119). EEUU: IEEE. doi: 10.1109/EDUCON.2011.5773286

Abbad, M. M., Morris, D., y de Nahlik, C. (2009). Looking under the bonnet: Factors affecting student adoption of E-learning systems in jordan. International Review of Research in Open and Distance Learning, 10(2), 1-15

Acarli, D. S., y Sağlam, Y. (2015). Investigation of pre-service teachers' intentions to use of social media in teaching activities within the framework of technology acceptance model. Procedia - Social and Behavioral Sciences, 176, 709-713. doi:http://dx.doi.org/10.1016/j.sbspro.2015.01.530

Ajzen, I. (1985). From intentions to actions: A theory of planned behavior. En J. Kuhl y J. Beckmann (Eds.), (pp. 11-39). Berlin Heidelberg: Springer. doi:10.1007/978-3642-69746-3_2

Al-Somali, S. A., Gholami, R., y Clegg, B. (2009). An investigation into the acceptance of online banking in Saudi Arabia. Technovation, 29(2), 130-141.

Resistencia al Cambio y Compatibilidad con las Tecnologías Móviles. Un Estudio Sobre la Intención de Uso Entre los Futuros Docentes. José Carlos Sánchez-Prieto, Susana Olmos-Migueláñez, Francisco J.

García-Peñalvo. 
Ariff, M. S. M., Yeow, S. M., y Zakuan, N. (2014). Acceptance of internet banking systems among young users; the effect of technology acceptance model. Advanced Science Letters, 20(1), 268-272.

Bachtiar, F. A., Rachmadi, A., y Pradana, F. (2014). Acceptance in the deployment of blended learning as a learning resource in information technology and computer science program, Brawijaya university Proceedings of the 2014 Asia-Pacific Conference on Computer Aided System Engineering (APCASE) (pp. 131-135). EEUU: IEEE. doi:10.1109/APCASE.2014.6924486

Bhattacherjee, A., y Hikmet, N. (2007). Physicians' resistance toward healthcare information technology: A theoretical model and empirical test. European Journal of Information Systems, 16(6), 725-737.

Biranvand, V. P., Hakkak, M., y Nejad, O. N. (2015). Resistance to change in online banking and extension technology acceptance model (TAM). Bulletin of the Georgian National Academy of Sciences, 9(1), 464-472.

Bourgonjon, J., De Grove, F., De Smet, C., Van Looy, J., Soetaert, R., y Valcke, M. (2013). Acceptance of game-based learning by secondary school teachers. $\begin{array}{llll}\text { Computers } \& \text { Education, 67, 21-35. } & \text {. }\end{array}$ doi:http://dx.doi.org/10.1016/j.compedu.2013.02.010

Briz-Ponce, L., y García-Peñalvo, F. J. (2015). An empirical assessment of a technology acceptance model for apps in medical education. Journal of Medical Systems, 39(11), 176-180. doi:http://doi.org/10.1007/s10916-015-0352-x

Cao Y., Ajjan, H., y Hong, P. (2013). Using social media applications for educational outcomes in college teaching: A structural equation analysis. British Journal of Educational Technology, 44(4), 581-593. doi:10.1111/bjet.12066

Cenfetelli, R. T. (2004). Inhibitors and enablers as dual factor concepts in technology usage. Journal of the Association for Information Systems, 5(11), 473-492.

Chang, S., y Tung, F. (2008). An empirical investigation of students' behavioural intentions to use the online learning course websites. British Journal of Educational Technology, 39(1), 71-83. doi:10.1111/j.1467-8535.2007.00742.x

Chen, F. H., Looi, C. K., y Chen, W. (2009). Integrating technology in the classroom: A visual conceptualization of teachers' knowledge, goals and beliefs. Journal of Computer Assisted Learning, 25(5), 470-488. doi:10.1111/j.13652729.2009.00323.x

Chen, J. (2011). The effects of education compatibility and technological expectancy on e-learning acceptance. Computers \& Education, 57(2), 1501-1511. doi:http://dx.doi.org/10.1016/j.compedu.2011.02.009

Resistencia al Cambio y Compatibilidad con las Tecnologías Móviles. Un Estudio Sobre la Intención de Uso Entre los Futuros Docentes. José Carlos Sánchez-Prieto, Susana Olmos-Migueláñez, Francisco J. 
Chen, Y., Lin, Y., Yeh, R. C., y Lou, S. (2013). Examining factors affecting college students' intention to use web-based instruction systems: Towards an integrated model. Turkish Online Journal of Educational Technology, 12(2), 111-121.

Cheng, F. P., Wang, K. H., y Lin, I. C. (2013). Measuring the adoption and resistance of elearning by students Proceedings of The International Conference on ETechnologies and Business on the Web (EBW2013) (pp. 247-249).

Cheng, Y. (2015). Towards an understanding of the factors affecting m-learning acceptance: Roles of technological characteristics and compatibility. Asia Pacific Management Review, 20(3), 109-119. doi:http://dx.doi.org/10.1016/j.apmrv.2014.12.011

Clarkson, D. B., y Jenrich, R. I. (1988). Quartic rotation criteria and algorithms. Psychometrika, 53(2), 251-259.

Davis, F. D. (1989). Perceived usefulness, perceived ease of use, and user acceptance of information technology. MIS Quarterly, 13(3), 319-340.

Davis, F. D., Bagozzi, R. P., \& Warshaw, P. R. (1989). User acceptance of computer technology: A comparison of two theoretical models. Management Science, 35(8), 982-1003. doi: $10.1287 / \mathrm{mnsc} .35 .8 .982$

Davis, F. D., y Venkatesh, V. (1996). A critical assessment of potential measurement biases in the technology acceptance model: Three experiments. International Journal of Human-Computer Studies, 45(1), 19-45. doi:http://dx.doi.org/10.1006/ijhc.1996.0040

Fishbein, M., y Ajzen, I. (1975). Belief, attitude, intention, and behavior: An introduction to theory and research. Reading, Massachusets: Addison-Wesley Pub. Co.

Fong, R. W., Lee, J. C., Chang, C., Zhang, Z., Ngai, A. C., y Lim, C. P. (2014). Digital teaching portfolio in higher education: Examining colleagues' perceptions to inform implementation strategies. Internet and Higher Education, 20, 60-68.

Fornell, C., Tellis, G. J., y Zinkhan, G. M. (1982). Validity assessment: A structural equations approach using partial least squares. En B. J. Walker (Ed.), An assessment of marketing thought and practice (pp. 405-409). Chicago: American Marketing Association.

Fornell, C., y Larcker, D. F. (1981). Evaluating structural equation models with unobservable variables and measurement error. Journal of Marketing Research, 18(1), 39-50. doi:10.2307/3151312

García-Peñalvo, F. J., y Seoane-Pardo, A. M. (2015). Una revisión actualizada del concepto de eLearning. Décimo Aniversario. Education in the Knowledge Society, 16(1), 119-144. doi:10.14201/eks2015161119144

Resistencia al Cambio y Compatibilidad con las Tecnologías Móviles. Un Estudio Sobre la Intención de Uso Entre los Futuros Docentes. José Carlos Sánchez-Prieto, Susana Olmos-Migueláñez, Francisco J. 
Gagnon, M., Ghandour, E. K., Talla, P. K., Simonyan, D., Godin, G., Labrecque, M., y Rousseau, M. (2014). Electronic health record acceptance by physicians: Testing an integrated theoretical model. Journal of Biomedical Informatics, 48, 17-27. doi:http://dx.doi.org/10.1016/j.jbi.2013.10.010

Goodhue, D. L., \& Loiacono, E. T. (2002). Randomizing survey question order vs. grouping questions by construct: An empirical test of the impact on apparent reliabilities and links to related constructs Proceedings of the 35th Annual Hawaii International Conference on System Sciences, 2002. HICSS (pp. 3456-3465). EEUU: IEEE. doi:10.1109/HICSS.2002.994385

Guo, X., Sun, Y., Wang, N., Peng, Z., y Yan, Z. (2013). The dark side of elderly acceptance of preventive mobile health services in china. Electronic Markets, 23(1), 49-61. doi:10.1007/s12525-012-0112-4

Hair, J. F., Black, W. C., Babin, B. J., Anderson, R. E., y Tatham, R. L. (2006). Multivariate data analysis (6th ed.). Nueva Jersey: Prentice-Hall International.

Hernández García, Á. (2008). Desarrollo de un modelo unificado de adopción del comercio electrónico entre empresas y consumidores finales. Aplicación al mercado español. E.T.S.I. Telecomunicación (UPM), Madrid.

Hsieh, P.-J. (2015). Healthcare professionals' use of health clouds: Integrating technology acceptance and status quo bias perspectives. International Journal of Medical Informatics, 84(7), 512-523. doi:http://dx.doi.org/10.1016/j.ijmedinf.2015.03.004

Hsieh, P.-J., Lai, H.-M., y Hong, Y.-L. (2015). Explaining Physicians' Acceptance and Resistance to the NHI Pharmacloud: A Theoretical Model and Empirical Test. En A. Kankanhalli, A. Burton-Jones, \& T. S. H. Teo (Eds.), PACIS 2015 Proceedings (pp. 247-263).

Hsieh, P.-J., Lai, H.-M., y Ye, Y. (2014). Patients' acceptance and resistance toward the health cloud: An integration of technology acceptance and status quo bias perspectives. En Proceedings of the 18th Pacific Asia Conference on Information Systems, (PACIS), Chengdou, China (pp. 230-245).

Huang, H., Liaw, S., y Lai, C. (2013). Exploring learner acceptance of the use of virtual reality in medical education: A case study of desktop and projection-based display systems. Interactive Learning Environments, 24(1), 3-19. doi:10.1080/10494820.2013.817436

Hung, H., y Cho, V. (2008). Continued usage of e-learning communication tools: A study from the learners' perspective in Hong Kong. International Journal of Training and Development, 12(3), 171-187. doi:10.1111/j.14682419.2008.00302.x

Resistencia al Cambio y Compatibilidad con las Tecnologías Móviles. Un Estudio Sobre la Intención de Uso Entre los Futuros Docentes. José Carlos Sánchez-Prieto, Susana Olmos-Migueláñez, Francisco J. 
Islam, A. K. M. N. (2013). Investigating e-learning system usage outcomes in the university context. Computers \& Education, 69, 387-399. doi:http://dx.doi.org/10.1016/j.compedu.2013.07.037

John, S. P. (2015). The integration of information technology in higher education: A study of faculty's attitude towards IT adoption in the teaching process. Contaduría y Administración, $60 \quad$ (Supplement 1), 230-252. doi:http://dx.doi.org/10.1016/j.cya.2015.08.004

Karahanna, E., Agarwal, R., y Angst, C. M. (2006). Reconceptualizing compatibility beliefs in technology acceptance research. MIS Quarterly, 30(4), 781-804.

King, W. R., y He, J. (2006). A meta-analysis of the technology acceptance model. Information \& Management, 43(6), 740-755. doi:http://dx.doi.org/10.1016/j.im.2006.05.003

Klem, L. (2000). Structural equation modelling. En L. Grimm, y P., Yarnold (Eds.), Reading and understanding multivariate statistics (pp. 227-260). Washington, D.C.: American Psychological Association.

Kline, R. B. (2005). Principles and practice of structural equation modelling. New York: Guilford Press.

Lee, Y., Hsieh, Y., y Hsu, C. (2011). Adding innovation diffusion theory to the technology acceptance model: Supporting employees' intentions to use E-learning systems. Educational Technology \& Society, 14(4), 124-137.

Lei, J. (2009). Digital natives as preservice teachers: What technology preparation is needed? Journal of Computing in Teacher Education, 25(3), 87-97.

Lewin, K. (1947). Frontiers in group dynamics: Concept, method, and reality in social sciences, social equilibria, and social change. Human Relations, 1(1), 5-41.

Liao, H., y Lu, H. (2008). Richness versus parsimony antecedents of technology adoption model for e-learning websites. En F. Li, J. Zhao, T. K. Shih, R. L. Q. Li \& D. McLeod (Eds.), Advances in web based learning - ICWL 2008 (pp. 8-17). Berlin: Springer.

Lloret-Segura, S., Ferreres-Traver, A., Hernández-Baeza, A., y Tomás-Marco, I. (2014). El análisis factorial exploratorio de los ítems: Una guía práctica, revisada y actualizada. Anales De Psicología, 30(3), 1151-1169.

Ma, C., Chao, C., y Cheng, B. (2013). Integrating technology acceptance model and task-technology fit into blended E-learning system. Journal of Applied Sciences, 13(5), 736-742.

McDonald, R. P., y Ho, M. R. (2002). Principles and practice in reporting structural equation analyses. Psychological Methods, 7(1), 64-82.

Resistencia al Cambio y Compatibilidad con las Tecnologías Móviles. Un Estudio Sobre la Intención de Uso Entre los Futuros Docentes. José Carlos Sánchez-Prieto, Susana Olmos-Migueláñez, Francisco J.

García-Peñalvo. 
Mejia, C., y Phelan, K. V. (2014). Academic referent group influences on hospitality students' intentions to enroll in an online course. Journal of Hospitality \& Tourism Education, 26(2), 65-74. doi:10.1080/10963758.2014.900378

Moore, G. C., y Benbasat, I. (1991). Development of an instrument to measure the perceptions of adopting an information technology innovation. Information Systems Research, 2(3), 192-222. doi:10.1287/isre.2.3.192

Nov, O., y Schecter, W. (2012). Dispositional resistance to change and hospital physicians' use of electronic medical records: A multidimensional perspective. Journal of the American Society for Information Science and Technology, 63(4), 648-656. doi:10.1002/asi.22602

Nov, O., y Ye, C. (2009). Resistance to change and the adoption of digital libraries: An integrative model. Journal of the American Society for Information Science and Technology, 60(8), 1702-1708. doi:10.1002/asi.21068

Padilla-Meléndez, A., del Aguila-Obra, A. R., y Garrido-Moreno, A. (2013). Perceived playfulness, gender differences and technology acceptance model in a blended learning scenario. Computers \& Education, 63, 306-317. doi:http://dx.doi.org/10.1016/j.compedu.2012.12.014

Park, S. Y. (2009). An analysis of the technology acceptance model in understanding university students' behavioral intention to use e-learning. Educational Technology \& Society, 12(3), 150-162.

Parra-Meroño, M. C., y Carmona-Martínez, M. M. (2011). Las tecnologías de la información y las comunicaciones en la enseñanza superior española. factores explicativos del uso del campus virtual. Estudios Sobre Educación, 20, 73-98.

Ramírez-Correa, P., Rondán-Cataluña, F. J., y Arenas-Gaitán, J. (2010). Influencia del género en la percepción y adopción de e-learning: Estudio exploratorio en una universidad chilena. Journal of Technology Management and Innovation, 5(3), 129-141.

Rienties, B., Giesbers, B., Lygo-Baker, S., Ma, H. W. S., \& Rees, R. (2016). Why some teachers easily learn to use a new virtual learning environment: A technology acceptance perspective. Interactive Learning Environments, 24(3), 539-552. doi:10.1080/10494820.2014.881394

Rogers, E. M. (1962). Diffusion of innovations. New York: Free Press of Glencoe.

Sánchez-Franco, M. J. (2010). WebCT - the quasimoderating effect of perceived affective quality on an extending technology acceptance model. Computers and Education, 54(1), 37-46.

Sánchez-Franco, M. J., Martínez-López, F. J., y Martín-Velicia, F. A. (2009). Exploring the impact of individualism and uncertainty avoidance in web-based electronic

Resistencia al Cambio y Compatibilidad con las Tecnologías Móviles. Un Estudio Sobre la Intención de Uso Entre los Futuros Docentes. José Carlos Sánchez-Prieto, Susana Olmos-Migueláñez, Francisco J.

García-Peñalvo.

Página 31 de 31 
learning: An empirical analysis in european higher education. Computers and Education, 52(3), 588-598.

Sánchez Prieto, J. C., Olmos Migueláñez, S., y García-Peñalvo, F. J. (2014). Understanding mobile learning: Devices, pedagogical implications and research lines. Revista Teoría de la Educación: Educación y Cultura en la Sociedad de la Información, 15(1), 20-42.

Sánchez Prieto, J. C., Olmos Migueláñez, S., \& García-Peñalvo, F. J. (2016). Informal Tools in Formal Contexts: Development of a Model to Assess the Acceptance of Mobile Technologies among Teachers. Computers in Human Behavior, 55A, 519528. doi:http://dx.doi.org/10.1016/j.chb.2015.07.002

Schoonenboom, J. (2014). Using an adapted, task-level technology acceptance model to explain why instructors in higher education intend to use some learning management system tools more than others. Computers and Education, 71, 247256.

Šebjan, U., y Tominc, P. (2015). Impact of support of teacher and compatibility with needs of study on usefulness of SPSS by students. Computers in Human Behavior, 53, 354-365. doi:http://dx.doi.org/10.1016/j.chb.2015.07.022

Stols, G., y Kriek, J. (2011). Why don't all maths teachers use dynamic geometry software in their classrooms? Australasian Journal of Educational Technology, 27(1), 137-151.

Sun, Y., Bhattacherjee, A., y Ma, Q. (2009). Extending technology usage to work settings: The role of perceived work compatibility in ERP implementation. Information \& Management, 46(6), 351-356. doi:http://dx.doi.org/10.1016/j.im.2009.06.003

Teo, T. (2012). Examining the intention to use technology among pre-service teachers: An integration of the technology acceptance model and theory of planned behavior. Interactive Learning Environments, 20(1), 3-18. doi:10.1080/10494821003714632

Teo, T., Lee, C. B., Chai, C. S., y Wong, S. L. (2009). Assessing the intention to use technology among pre-service teachers in singapore and malaysia: A multigroup invariance analysis of the technology acceptance model (TAM). Computers \& Education, $\quad$ 53(3), 1000-1009. doi:http://dx.doi.org/10.1016/j.compedu.2009.05.017

Teo, T., y Noyes, J. (2011). An assessment of the influence of perceived enjoyment and attitude on the intention to use technology among pre-service teachers: A structural equation modeling approach. Computers \& Education, 57(2), 16451653. doi:http://dx.doi.org/10.1016/j.compedu.2011.03.002

Resistencia al Cambio y Compatibilidad con las Tecnologías Móviles. Un Estudio Sobre la Intención de Uso Entre los Futuros Docentes. José Carlos Sánchez-Prieto, Susana Olmos-Migueláñez, Francisco J. 
Tornatzky, L. G., y Klein, K. J. (1982). Innovation characteristics and innovation adoption-implementation: A meta-analysis of findings. IEEE Transactions On Engineering Management, EM-29(1), 28-45. doi:10.1109/TEM.1982.6447463

Valtonen, T., Kukkonen, J., Kontkanen, S., Sormunen, K., Dillon, P., y Sointu, E. (2015). The impact of authentic learning experiences with ICT on pre-service teachers' intentions to use ICT for teaching and learning. Computers \& Education, 81, 49-58. doi:http://dx.doi.org/10.1016/j.compedu.2014.09.008

Venkatesh, V., y Davis, F. D. (2000). A theoretical extension of the technology acceptance model: Four longitudinal field studies. Management Science, 46(2), 186-204.

Venkatesh, V., y Bala, H. (2008). Technology acceptance model 3 and a research agenda on interventions. Decision Sciences, 39(2), 273-315. doi:10.1111/j.15405915.2008.00192.x

Venter, P., van Rensburg, M. J., y Davis, A. (2012). Drivers of learning management system use in a south african open and distance learning institution. Australasian Journal of Educational Technology, 28(2), 183-198.

Wang, W., y Wang, C. (2009). An empirical study of instructor adoption of web-based learning systems. Computers \& Education, 53(3), 761-774. doi:http://dx.doi.org/10.1016/j.compedu.2009.02.021

Wu, C., y Liu, C. (2013). Acceptance of ICT-mediated teaching/learning systems for elementary school teachers: Moderating effect of cognitive styles. Education and Information Technologies, 20(2), 381-401. doi:10.1007/s10639-013-9290-8

Zhang, W., y Xu, P. (2011). Do I have to learn something new? mental models and the acceptance of replacement technologies. Behaviour \& Information Technology, 30(2), 201-211. doi:10.1080/0144929X.2010.489665

Resistencia al Cambio y Compatibilidad con las Tecnologías Móviles. Un Estudio Sobre la Intención de Uso Entre los Futuros Docentes. José Carlos Sánchez-Prieto, Susana Olmos-Migueláñez, Francisco J. 